Check for updates

Cite this: Chem. Sci., 2019, 10, 6683

๑ All publication charges for this article have been paid for by the Royal Society of Chemistry

Received 13th March 2019

Accepted 28th May 2019

DOI: $10.1039 / c 9 s c 01233 h$

rsc.li/chemical-science

\section{Sacrificial oxidation of a self-metal source for the \\ Sacrificial oxidation of a self-metal source for the
rapid growth of metal oxides on quantum dots towards improving photostability $\uparrow$}

\author{
Lu Huang, ${ }^{\text {ab }}$ Zhichun Li, ${ }^{a}$ Congyang Zhang, (D) a Long Kong, ${ }^{a}$ Bo Wang, ${ }^{a}$ \\ Shouqiang Huang, (D) a Vaishali Sharma, ${ }^{a}$ Houyu Ma, ${ }^{c}$ Qingchen Yuan, ${ }^{c}$ Yue Liu, ${ }^{c}$ \\ Guoqing Shen, ${ }^{b}$ Kaifeng Wu (D) d and Liang Li (iD *a
}

Growth of metal oxide layers on quantum dots (QDs) has been regarded as a good way to improve the photostability of QDs. However, direct growth of metal oxides on individual QD remains a great challenge. Here we report a novel approach to rapidly anchor metal oxides on QD surfaces through a sacrificial oxidation of a self-metal source strategy. As typical core/shell QDs, CdSe/CdS or aluminum doped CdSe/CdS (CdSe/CdS:Al) QDs were chosen and treated with peroxide (benzoyl peroxide). Selfmetal sources (cadmium or/and aluminum) can be easily sacrificially oxidized, leading to the quick growth of cadmium oxide $(\mathrm{CdO})$ or aluminum/cadmium hybrid oxides $\left(\mathrm{Al}_{2} \mathrm{O}_{3} / \mathrm{CdO}\right)$ on the surface of individual $\mathrm{QD}$ for improved photostability. Compared with $\mathrm{CdO}, \mathrm{Al}_{2} \mathrm{O}_{3}$ possesses excellent barrier properties against moisture and oxygen. Therefore, $\mathrm{CdSe} / \mathrm{CdS}$ QDs with the protection of an $\mathrm{Al}_{2} \mathrm{O}_{3} / \mathrm{CdO}$ hybrid layer show much superior photostability. Under strong illumination with blue light, the QDs coated with the $\mathrm{Al}_{2} \mathrm{O}_{3} / \mathrm{CdO}$ hybrid layer retained $100 \%$ of the original photoluminescence intensity after $70 \mathrm{~h}$, while that of the untreated CdSe/CdS:Al, the treated CdSe/CdS and the CdSe/CdS QDs dropped to $65 \%, 45 \%$, and $5 \%$, respectively. Furthermore, we demonstrate that this method can be extended to other metal-doped QD systems, even including some inactive metals difficult to be oxidized spontaneously in an ambient atmosphere, which provides a new way to stabilize QDs for diverse optoelectronic applications. optoelectronic applications.

\section{Introduction}

Semiconductor quantum dots (QDs) have been intensively studied over the past few decades because of their wide range of applications such as in single-photon sources, ${ }^{1-3}$ light-emitting diodes, ${ }^{4-6}$ solar cells, ${ }^{7-9}$ and biomedical labeling. ${ }^{10,11}$ Nevertheless, the practical applications of QDs are severely restricted due to their poor stability against air and moisture. To date, many approaches have been developed to improve the chemical/ photostability of QDs, such as core-shell structure construction, ${ }^{12-14}$ silica coating, ${ }^{15}$ polymer encapsulation, ${ }^{16}$ and embedding into salt crystals ${ }^{17}$ or siloxane films. ${ }^{18}$ Metal oxides such as

${ }^{a}$ School of Environmental Science and Engineering, Shanghai Jiao Tong University, 800 Dongchuan Road, Shanghai 200240, China.E-mail: liangli117@sjtu.edu.cn

${ }^{b}$ School of Agriculture and Biology, Shanghai Jiao Tong University, 800 Dongchuan Road, Shanghai 200240, China

${ }^{c}$ School of Materials Science and Engineering, Shanghai Jiao Tong University, 800 Dongchuan Road, Shanghai 200240, China

${ }^{d}$ Dalian Institute of Chemical Physics, Chinese Academy of Sciences, 457 Zhongshan Road, Dalian 116021, China

$\dagger$ Electronic supplementary information (ESI) available: Experimental section, characterization details and additional figures and tables. See DOI: 10.1039/c9sc01233h aluminum oxide $\left(\mathrm{Al}_{2} \mathrm{O}_{3}\right)$, titanium oxide, and zirconium oxide are often used for coating on metal, organic optoelectronic devices, and phosphors for protecting them from oxidation and corrosion, due to their excellent barrier properties against moisture and oxygen. ${ }^{19-21}$ However, direct growth of metal oxides on individual QD still remains a great challenge.

It is well known that zinc blende CdSe QDs possess higher photostability than wurtzite CdSe QDs under ambient conditions, because a thin layer of CdO can be formed on the surface of zinc blende CdSe QDs through the oxidization of $\mathrm{Cd}$ in air. ${ }^{22}$ Besides, in our previous work, we demonstrated that aluminum (Al) can be successfully doped into the QDs to enhance the photostability, since $\mathrm{Al}$ in the shell can also be oxidized to $\mathrm{Al}_{2} \mathrm{O}_{3}$ in air and serve as a self-passivation layer. However, these spontaneous oxidation processes are usually very slow and the oxide layer may be loose; thus they are adverse to practical applications. In addition, as we all know, in the metallurgy industry, a dense metal oxide protective layer can always be quickly generated on metal surfaces by immersion in strong oxidizers (such as nitric acid, ${ }^{23}$ sulfuric acid $^{24}$ and peroxides ${ }^{25}$ ). Inspired by these findings, we envisage that inherent metals in QDs can be employed for rapidly generating a metal oxide layer on the surface of QDs through a proper oxidizing agent. 
Accordingly, herein we developed a sacrificial oxidation of a selfmetal source (SOSMS) strategy to grow metal oxides on individual QD surfaces for protecting them from corrosion with high stability. Considering the strong acidity of nitric acid and sulfuric acid, which may etch the QDs, our study here focuses on benzoyl peroxide $\left(\left(\mathrm{C}_{6} \mathrm{H}_{5} \mathrm{CO}\right)_{2} \mathrm{O}_{2}\right.$, BPO), which is a typical peroxide and often used as a radical initiator to induce polymerization in polymer synthesis. BPO has a weak oxygen-oxygen bond which can readily break and release a benzoic acid radical with high reactivity to promote the polymerization or oxidation processes. $^{26-28}$ Moreover, BPO is easily dissolved into organic nonpolar solvents, making it compatible with colloidal QD systems dispersed in toluene or hexane. The whole process can be described using a simple model and is shown in Fig. 1. Firstly, two typical core/shell QDs, CdSe/CdS and aluminum doped CdSe/ CdS (CdSe/CdS:Al) QDs, were chosen as our target research systems. A CdS shell was epitaxially grown on the CdSe core without or with the doping of $\mathrm{Al}$, and then treated with BPO, which behaves as an appropriate oxidizer and releases benzoic acid radicals (Fig. $\mathrm{S} 1 \dagger$ ) to promote the transformation from $\mathrm{Al}-\mathrm{S} /$ $\mathrm{Cd}-\mathrm{S}$ to $\mathrm{Al}-\mathrm{O} / \mathrm{Cd}-\mathrm{O}$, finally leading to the formation of a robust $\mathrm{CdO}$ or $\mathrm{Al}_{2} \mathrm{O}_{3} / \mathrm{CdO}$ hybrid oxide protective layer on the $\mathrm{CdSe} / \mathrm{CdS}$ and CdSe/CdS:Al QD surfaces, respectively, for significantly enhanced chemical/photostability. In addition, we proved that this method could be extended to other metal-doped QD systems, such as CdSe/CdS:Cr QDs, CdSe/CdS:Zr QDs and CuInS ${ }_{2} / \mathrm{ZnS}: \mathrm{Al}$ (CIS/ZnS:Al) QDs. Importantly, peroxide (such as BPO) treatment can promote the oxidation of metal on QD surfaces for improved photostability regardless of the reactivity of the individual metal, even including some inactive metals difficult to be oxidized spontaneously in an ambient atmosphere.

\section{Results and discussion}

\section{SOSMS strategy for CdSe/CdS QDs}

Firstly, we choose CdSe/CdS core/shell QDs as a model system. To perform the experiment of peroxide treatment, BPO (in toluene, $0.01 \mathrm{M}, 100 \mu \mathrm{L}$ ) was slowly added into a CdSe/CdS QD solution $\left(15 \mathrm{mg} \mathrm{mL}^{-1}, \mathrm{Abs}_{600 \mathrm{~nm}}=2\right)$ and this solution was kept at $60{ }^{\circ} \mathrm{C}$ for $2 \mathrm{~h}$. After treatment, the QDs were purified and redispersed in toluene for further characterization. The transmission electron microscope (TEM) images of the CdSe/CdS QDs before and after BPO treatment are shown in Fig. S2. $\dagger$

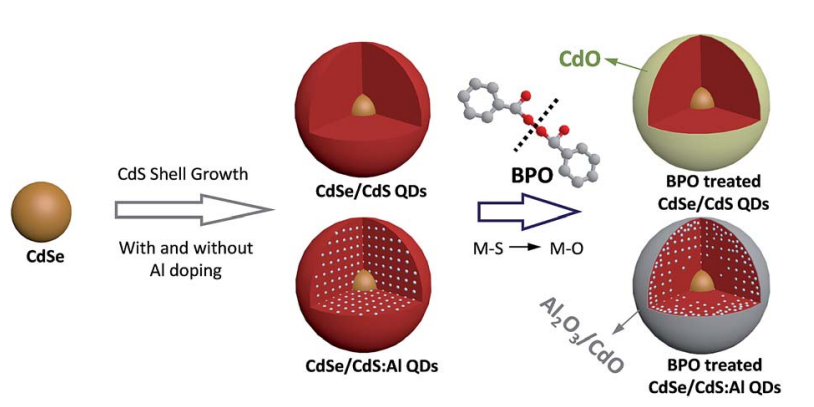

Fig. 1 Schematic of anchoring $\mathrm{CdO}$ and $\mathrm{Al}_{2} \mathrm{O}_{3} / \mathrm{CdO}$ hybrid oxide protective layers on the $\mathrm{CdSe} / \mathrm{CdS}$ and CdSe/CdS:Al QDs, respectively.
Compared with the original QDs, after BPO treatment, the CdSe/CdS QD sample exhibited more uniform distribution. The average size slightly decreased from $9.9 \mathrm{~nm}$ to $9.4 \mathrm{~nm}$. The optical spectra, shown in Fig. 2a, reveal that both the absorption and PL spectra exhibited a slight blueshift by about $3 \mathrm{~nm}$. Furthermore, the photostability of $\mathrm{CdSe} / \mathrm{CdS}$ QDs was measured on a blue LED module (details are given in the ESI $\dagger$ ). The results showed that the photostability of CdSe/CdS QDs was indeed improved after BPO treatment (Fig. 2b). After $70 \mathrm{~h}$ of blue light irradiation, the BPO treated CdSe/CdS QDs retained $45 \%$ of the original PL intensity, while the value for the untreated CdSe/CdS QDs was only 5\%. For detailed analysis, the X-ray photoelectron spectroscopy (XPS) data of the CdSe/CdS QDs are shown in Fig. S3. $\dagger$ We observed that the peaks of both Cd 3d and O 1s shifted to a lower binding energy after BPO treatment. In addition, the $\mathrm{O}$ content increased 1.9 times compared to its initial value (Table $\mathrm{S} 1 \dagger$ ). All these results suggested that the $\mathrm{Cd}-\mathrm{S}$ bonds were likely to partly convert into $\mathrm{Cd}-$ $\mathrm{O}$ and quickly form CdO on the surface of CdSe/CdS QDs. ${ }^{29,30}$ However, the photostability improvement was still not satisfactory (Fig. 2b), perhaps because the CdO layer on the surface did not provide enough protection to QDs.

\section{SOSMS strategy for CdSe/CdS:Al QDs}

Back to the metallurgy science, Al alloying has often been applied to improve the anti-corrosion properties of $\mathrm{Zn}$ or $\mathrm{Cd}$ metal because $\mathrm{Al}$ easily forms an $\mathrm{Al}_{2} \mathrm{O}_{3}$ layer on the metal surface
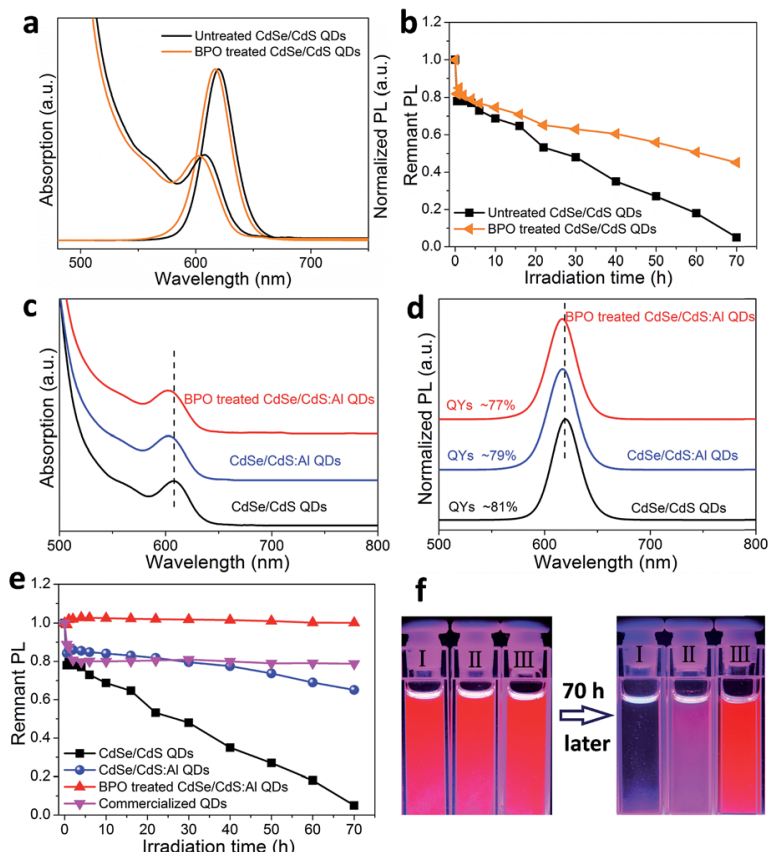

Fig. 2 The optical properties of QDs before and after BPO treatment. (a) Absorption and PL spectra and (b) photostability of the untreated and BPO treated CdSe/CdS QDs. (c) Absorption and (d) PL spectra of CdSe/CdS, CdSe/CdS:Al, and BPO treated CdSe/CdS:Al QDs. (e) Photostability of CdSe/CdS, CdSe/CdS:Al, BPO treated CdSe/CdS:Al, and commercialized QDs $\left(25^{\circ} \mathrm{C}, 60 \% \mathrm{RH}\right)$. (f) The optical images of CdSe/CdS QDs (I), CdSe/CdS:Al QDs (II), and BPO treated CdSe/CdS:Al QDs (III) before and after $70 \mathrm{~h}$ light irradiation (175 mW cm${ }^{-2}, 450 \mathrm{~nm}$ ). 
with better protective ability than the $\mathrm{Zn}$ or Cd oxides. ${ }^{\mathbf{3 1}}$ Here, with the aim of better stability, we tried to dope $\mathrm{Al}$ into CdSe/CdS QDs, and then expected rapid growth of an $\mathrm{Al}_{2} \mathrm{O}_{3}$ protective layer on QD surfaces by sacrificial oxidation of inherent Al. CdSe/CdS:Al QDs were prepared by a modified procedure reported previously. ${ }^{32}$ TEM images of CdSe/CdS:Al QDs (Fig. S4 $\dagger$ ) show that the Al doping does not generate any byproducts, but slightly changes the morphology and reduces the size of $\mathrm{CdSe} / \mathrm{CdS}$ QDs. From X-ray powder diffraction (XRD) patterns (Fig. S5 $\dagger$ ), we can confirm that the CdSe/ CdS QD sample exhibits a zinc-blende phase of CdS with three peaks assigned to (111), (220), and (311). After Al doping, the position of these peaks slightly shifts to a larger angle, which could be attributed to the doping of smaller $\mathrm{Al}$ atoms into the CdS shell. ${ }^{33}$ In general, impurity doping will generate some defects and thus affect the optical properties of QDs. The average PL lifetimes were $38.5 \mathrm{~ns}$ and $35.5 \mathrm{~ns}$ for CdSe/CdS and CdSe/CdS:Al QDs (Fig. S6†), respectively, which was in line with the variation of PLQYs (81\% and $79 \%$ for CdSe/CdS and CdSe/CdS:Al QDs, respectively). These results indicated that a few defects were generated during the process of Al doping, but they didn't significantly affect the optical properties of QDs. This phenomenon can be explained by the fact that the electronic energy level of doped $\mathrm{Al}$ is above the conduction band of the CdS shell of CdSe/CdS QDs, and thus the $\mathrm{Al}$ is not involved in the exciton behavior of the CdSe/CdS QDs. ${ }^{34}$ Energydispersive X-ray spectroscopy (EDX) and Inductively coupled plasma emission spectrometry (ICP) results indicate the existence of $\mathrm{Al}$ atoms in $\mathrm{QDs}$, and the $\mathrm{Al} / \mathrm{Cd}$ molar ratios are 0.085 and 0.110 , respectively. The successful doping of $\mathrm{Al}$ was further confirmed using high-angle annular dark field scanning transmission electron microscope (HAADF-STEM) images and elemental maps. We could control the doping location, for example, the $\mathrm{Al}$ atoms could be purposely doped only into the center of QDs (the details are shown in Fig. S7†).
As expected, the existing $\mathrm{Al}$ in the $\mathrm{CdS}$ shell led to a significant difference in the behavior of QDs after BPO treatment. Firstly, the size and optical properties did not show any obvious change (Fig. S8† and $2 \mathrm{c}$ and d), which was different from the case of CdSe/CdS QDs: the size decreased (Fig. S2 $\dagger$ ) and optical spectra showed a blue shift (Fig. 2a) after BPO treatment. Secondly, the photostability of CdSe/CdS:Al QDs remarkably improved (Fig. 2e). After $70 \mathrm{~h}$ blue light irradiation, the PL intensity of the BPO treated CdSe/CdS:Al QDs remained unchanged $(100 \%)$, and was much higher than that of the untreated CdSe/CdS:Al QDs (dropped to 65\%), the treated CdSe/ CdS QDs (dropped to 45\%, Fig. 2b), and even the commercialized QDs (dropped to 78\%). Furthermore, when we conducted an accelerated photostability test under harsher conditions $\left(85{ }^{\circ} \mathrm{C}, 85 \% \mathrm{RH}\right)$, the BPO treated CdSe/CdS:Al QD film exhibited the best photostability, and its PL remained almost unchanged after $200 \mathrm{~h}$ of operation (Fig. S9†). As shown in Fig. 2f, after $70 \mathrm{~h}$ light irradiation the BPO treated CdSe/CdS:Al QDs retained the same bright red emission, while the untreated CdSe/CdS:Al QDs and pristine CdSe/CdS QDs were severely faded and completely lost their emission, respectively. This obviously indicates that the BPO treatment produced a more robust protective layer to dramatically improve the photostability of CdSe/CdS:Al QDs. Nuclear magnetic resonance quantification confirmed that the oleic acid ligands remained on QDs after BPO treatment (Fig. S10 $\dagger$ ), which ensured that the BPO treated QDs maintained good colloidal stability.

To demonstrate the generation of the protective layer on CdSe/CdS:Al QDs after BPO treatment, several characterization studies were conducted. No evident changes were found in the XRD pattern (Fig. S11 $\dagger$ ). The high-resolution TEM (HRTEM) images of the untreated and treated CdSe/CdS:Al QDs are shown in Fig. 3a and e. They clearly show shell-like structures
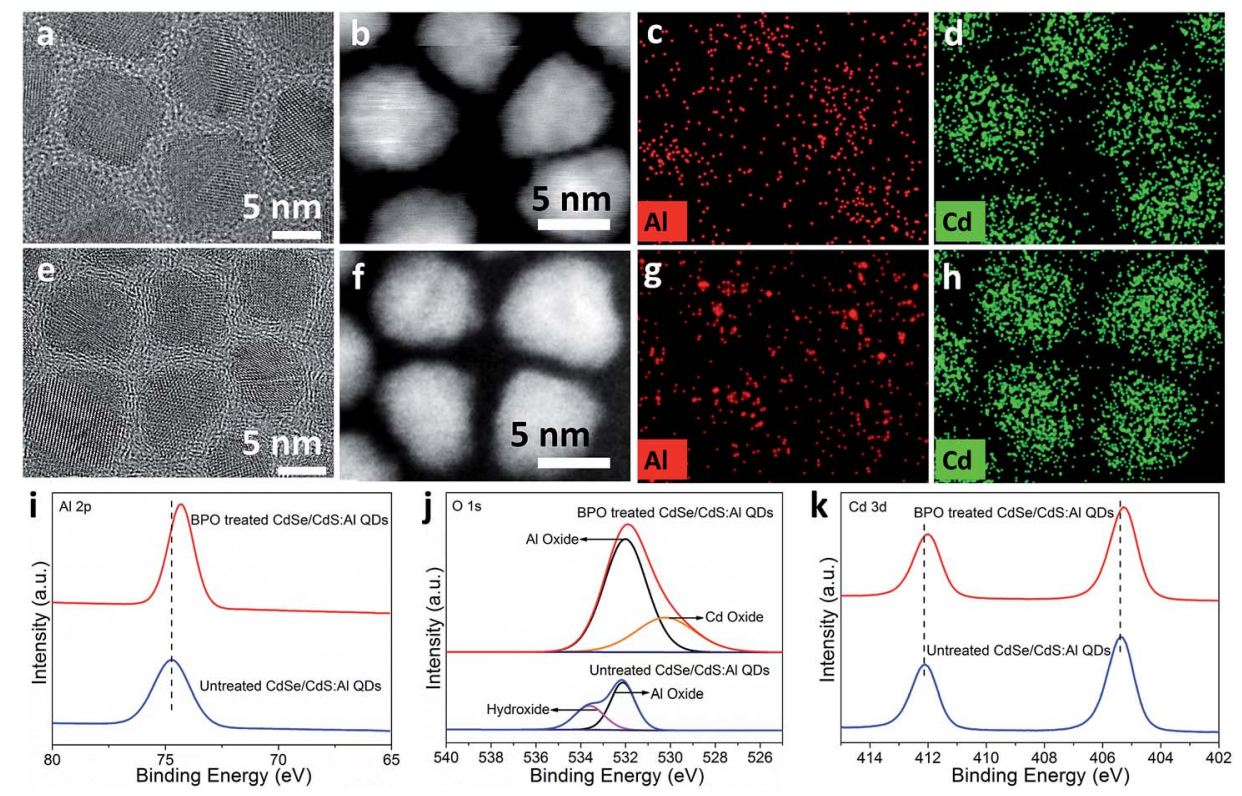

Fig. 3 Morphology and chemical state characterization of CdSe/CdS:Al QDs before and after BPO treatment. HRTEM images of the (a) untreated and (e) BPO treated CdSe/CdS:Al QDs. (b and f) HAADF-STEM images, the (c and g) Al and ( $d$ and h) Cd elemental maps of (b-d) untreated and ( $f-$ h) BPO treated CdSe/CdS:Al QDs. XPS spectra of (i) Al 2p, (j) O 1s, and (k) Cd 3d of CdSe/CdS:Al QDs before and after BPO treatment. 
on the surface of the treated sample. Moreover, we performed detailed HRTEM-FFT analysis on single QDs (Fig. S12 $\dagger$ ), which confirmed that this shell-like structure was an amorphous structure. The HAADF-STEM images and elemental maps of CdSe/CdS:Al QDs before and after BPO treatment are shown in Fig. $3 \mathrm{~b}-\mathrm{d}$ and $\mathrm{f}-\mathrm{h}$ and S13. $\dagger$ As expected, Cd (Fig. 3d), S (Fig.$\mathrm{S} 13 \mathrm{~d} \dagger$ ) and Se (Fig. S13e $\dagger$ ) elements have obvious elemental profiles before treatment, which almost remain the same after treatment. Comparatively, the Al profile is relatively indistinct owing to its lower content, and interestingly, the $\mathrm{Al}$ signals are aggregated into some circles around these QDs after BPO treatment. This change may be related to the formation of an $\mathrm{Al}-$ related amorphous layer around these QDs. But we still don't know what exactly it is and whether it completely covers the surface of QDs or not.

Furthermore, XPS characterization was performed. Fig. 3i shows the Al 2p XPS spectra of the untreated and treated CdSe/ CdS:Al QDs. The Al 2p peak of the untreated sample appeared at $74.70 \mathrm{eV}$ corresponding to $\mathrm{Al}-\mathrm{S}$ or $\mathrm{Al}-\mathrm{OH} .{ }^{35}$ After BPO treatment, the $\mathrm{Al} 2 \mathrm{p}$ peak shifted to a lower binding energy of $74.30 \mathrm{eV}$, which is generally attributed to Al-O. ${ }^{36}$ Therefore, we speculate that the chemical bonding of the $\mathrm{Al}$ element has changed during $\mathrm{BPO}$ treatment, possibly from $\mathrm{Al}-\mathrm{S}$ or $\mathrm{Al}-\mathrm{OH}$ to $\mathrm{Al}-\mathrm{O}$. As shown in Fig. 3j, the peak of $\mathrm{O} 1 \mathrm{~s}$ before BPO treatment could be divided into two peaks with binding energies of $532.1 \mathrm{eV}$ and $533.8 \mathrm{eV}$. These two peaks were assigned to the $\mathrm{Al}-\mathrm{O}$ bond of $\mathrm{Al}$ oxide and $\mathrm{Al}-\mathrm{OH}$ bond of $\mathrm{Al}$ hydroxide, ${ }^{37,38}$ respectively. It is highly possible that some $\mathrm{Al}$ atoms in CdSe/CdS:Al QDs have been already converted into $\mathrm{Al}$ hydroxides and $\mathrm{Al}$ oxides during the open-air purification process using acetone or alcohol (containing trace water). But it is worth pointing out that this kind of conversion occurred only for a small amount of $\mathrm{Al}$ atoms of QDs, because the oxygen content before treatment was quite low compared to the high oxygen content after treatment (Table $\mathrm{S} 2 \dagger)$. After treatment, the peak of $\mathrm{O}$ 1s was not perfectly symmetric (Fig. 3j). The main peak located at $532.0 \mathrm{eV}$ with a percentage of about $70 \%$ was assigned to the $\mathrm{Al}-\mathrm{O}$ bond in $\mathrm{Al}$ oxide. Meanwhile, considering the slight shift towards a relatively lower binding energy of the Cd $3 \mathrm{~d}$ peak after treatment (Fig. 3k), another shoulder peak centered at $530.2 \mathrm{eV}$ may be caused by the appearance of Cd oxide in the sample. ${ }^{29,30}$ These observations suggested that a certain $\mathrm{Cd}-\mathrm{S}$ component was converted into Cd-O as well, which co-existed with $\mathrm{Al}_{2} \mathrm{O}_{3}$ to form a hybrid oxide $\left(\mathrm{Al}_{2} \mathrm{O}_{3} / \mathrm{CdO}\right)$ protective layer on the surface of QDs after BPO treatment.

\section{Metal (Al) diffusion mechanism during the SOSMS process}

In order to further confirm the generation of the $\mathrm{Al}_{2} \mathrm{O}_{3} / \mathrm{CdO}$ layer on QD surfaces, an etching experiment following the ICP test was conducted on the untreated and BPO treated CdSe/CdS:Al QDs. The procedure of the etching experiment is provided in Fig. 4a. With the assistance of HF, the QDs were etched and peeled off layer by layer, leading to the shrinking of sizes (Fig. 4b) and gradual dissolution of $\mathrm{Cd}, \mathrm{S}$ and $\mathrm{Al}$ atoms into the solution. After etching, the QDs were precipitated by the anti-solvent acetone. Then the supernatants were collected to determine the elemental
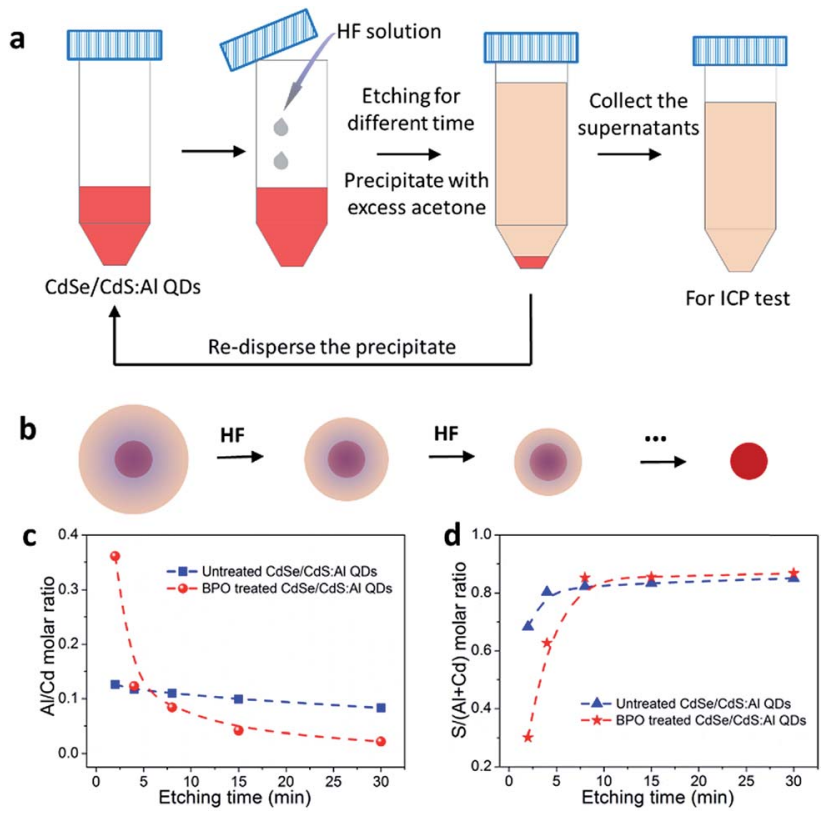

Fig. 4 The mechanism of Al diffusion during the SOSMS process. (a) Schematic of the etching experiment. (b) Evolution of the QDs etched by the HF solution at different times. (c) $\mathrm{Al} / \mathrm{Cd}$ and (d) $\mathrm{S} /(\mathrm{Al}+\mathrm{Cd}$ ) molar ratios of the supernatants for the untreated and BPO treated $\mathrm{CdSe} /$ CdS:Al QDs taken at different etching times according to the ICP test.

contents and the concentration by ICP testing, while the residue from QD precipitation was re-dispersed again for the subsequent etching step. Therefore, the ICP results of the supernatants for each etching step represent the elemental compositions of the peeled surface layers at different depths. As shown in Fig. S14a and $\mathrm{b}, \uparrow$ the absorption spectra of the untreated and treated CdSe/ CdS:Al QDs all underwent a blue shift to a shorter wavelength after etching step by step, suggesting the successful peeling of surface layers of CdSe/CdS:Al QDs. Obviously, as the etching continued, the ICP results shown in Fig. $4 \mathrm{c}$ and $\mathrm{d}$ reveal that the amount of $\mathrm{Al}$ in the BPO treated QD sample gradually decreased and the amount of $\mathrm{S}$ increased from the outer surface towards the interior, respectively. In contrast, for the untreated QD sample, the amount of these two elements remained constant with a small variation at different positions. These results strongly and directly confirm that the BPO treated QD sample has a more $\mathrm{Al}_{2} \mathrm{O}_{3}$ rich surface than the untreated one. Considering that in the initial peeled layer of the BPO treated CdSe/CdS:Al QDs a certain amount of $\mathrm{Cd}$ was also detected and both the $\mathrm{S} /(\mathrm{Al}+\mathrm{Cd})$ and S/Cd molar ratios (Fig. S15 $\dagger$ ) were much less than those of the untreated one, we should point out that it was indeed a hybrid $\mathrm{Al}_{2} \mathrm{O}_{3} / \mathrm{CdO}$ layer located on QD surfaces after BPO treatment, which was in accordance with the XPS results (Fig. $3 \mathrm{i}$ and $\mathrm{k}$ ).

In addition, the $\mathrm{Al} / \mathrm{Cd}$ molar ratio of the initially peeled layer (the first etching step, at the initial etching of $2 \mathrm{~min}$ ) was 0.361 for the BPO treated CdSe/CdS:Al QDs, which was much higher than that for the untreated sample (0.126). As the etching process continued, the $\mathrm{Al} / \mathrm{Cd}$ molar ratio decreased sharply. Even when the etching was over $8 \mathrm{~min}$, the $\mathrm{Al} / \mathrm{Cd}$ molar ratios of the peeled layers in BPO treated CdSe/CdS:Al QDs were less than 

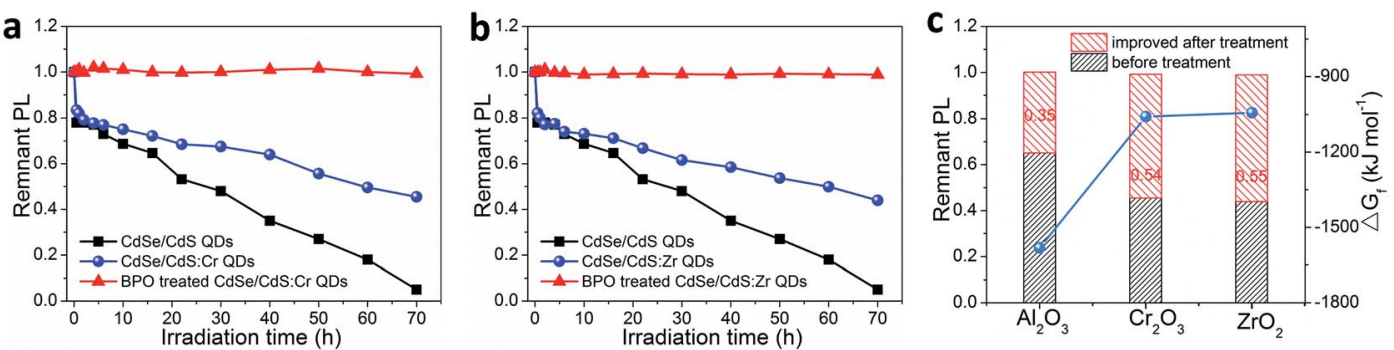

Fig. 5 The universality of the SOSMS strategy on improving the photostability of QDs. (a) Photostabilities of the CdSe/CdS, CdSe/CdS:Cr, and BPO treated CdSe/CdS:Cr QDs. (b) Photostabilities of the CdSe/CdS, CdSe/CdS:Zr, and BPO treated CdSe/CdS:Zr QDs. (c) Photostabilities of metal doped CdSe/CdS QDs before and after BPO treatment and Gibbs free energy of formation for selected metal oxides $\left(25{ }^{\circ} \mathrm{C}, 60 \% \mathrm{RH}\right)$.

those in the untreated QDs. This result clearly suggested that during the $\mathrm{BPO}$ treatment, some $\mathrm{Al}$ atoms in the deeper $\mathrm{CdS}$ shell of QDs possibly diffused from the inside towards the outside and accumulated on the QD surfaces to form oxides, thus boosting the photostability remarkably. Due to the selfpurification effect, as $\mathrm{Al}$ is a type of impurity atom, the doping of $\mathrm{Al}$ into our CdSe/CdS QDs is relatively difficult due to the reduced size, and the doped $\mathrm{Al}$ atoms could also be inclined to be expelled to the QD surface spontaneously, which can be ascribed to the high formation energy of impurities. In this case, the BPO treatment promoted the formation of $\mathrm{Al}_{2} \mathrm{O}_{3}$ on QD surfaces from the doped $\mathrm{Al}$ atoms, which may also provide a driving force to facilitate the self-purification effect, leading to the interesting $\mathrm{Al}$ diffusion phenomenon. ${ }^{39-41}$ This result further highlights the superiority of our strategy that a metal oxide (especially $\mathrm{Al}_{2} \mathrm{O}_{3}$ ) layer covering could be favorably generated on QD surfaces for significantly enhanced photostability, even with a relatively low doping level.

\section{Universality of the SOSMS strategy}

From the above results, we can conclude that $\mathrm{CdO}$ and $\mathrm{Al}_{2} \mathrm{O}_{3} / \mathrm{CdO}$ hybrid oxide protection layers can be generated on the surfaces of CdSe/CdS and CdSe/CdS:Al QDs after BPO treatment. In order to investigate the universality of our approach on improving the photostability of QDs, we applied it to other metal doped CdSe/CdS QD systems, such as CdSe/CdS:Cr and CdSe/CdS:Zr QDs. The absorption, PL spectra and photostability results are shown in Fig. S16 $\dagger$ and $5 \mathrm{a}$ and b, respectively. Similar to the Al doping, the doping of $\mathrm{Cr}$ and $\mathrm{Zr}$ resulted in a slight blueshift of absorption and PL spectra due to the partially suppressed crystal growth of QDs. And after BPO treatment, the optical spectra remained unchanged. Importantly, the photostabilities of both the CdSe/CdS:Cr and CdSe/CdS:Zr QDs improved significantly after doping and subsequent BPO treatment. Specifically, compared with the CdSe/CdS QDs, after $70 \mathrm{~h}$ blue light irradiation, the remnant PL intensities of CdSe/CdS:Al, CdSe/CdS:Cr, and CdSe/CdS:Zr QD samples (without BPO treatment) were $65 \%, 46 \%$, and $45 \%$, respectively, which indicated that the photostability of CdSe/CdS can also be improved by $\mathrm{Cr}$ and $\mathrm{Zr}$ doping, but to a lesser extent than by the $\mathrm{Al}$ doping. The spontaneous oxidation process of metals to form metal oxides is determined by the reactivity of the individual metal, or the reduction of Gibbs free energy $\left(\Delta G_{\mathrm{f}}\right)$. Considering that the $\Delta G_{\mathrm{f}}$ for the formation of $\mathrm{Al}_{2} \mathrm{O}_{3}\left(-1582.3 \mathrm{~kJ} \mathrm{~mol}^{-1}\right)$ was more negative than that of $\mathrm{Cr}_{2} \mathrm{O}_{3}\left(-1058.1 \mathrm{~kJ} \mathrm{~mol}^{-1}\right)$ and $\mathrm{ZrO}_{2}$ $\left(-1042.8 \mathrm{~kJ} \mathrm{~mol}^{-1}\right)$, the less pronounced photostability improvement of $\mathrm{Cr}$ and $\mathrm{Zr}$ doped $\mathrm{CdSe} / \mathrm{CdS}$ than the $\mathrm{Al}$ doped one can be attributed to the more difficult oxidation process of $\mathrm{Cr}$ and $\mathrm{Zr}$ than of $\mathrm{Al}^{42,43}$ However, after BPO treatment, the $\mathrm{Al}, \mathrm{Cr}$, and $\mathrm{Zr}$ doped CdSe/CdS QD samples showed significantly enhanced photostability and they all retained $100 \%$ of their original PL intensity after $70 \mathrm{~h}$ of blue light irradiation (Fig. 5c). These results clearly underlined that peroxide (such as $\mathrm{BPO}$ ) treatment can promote the oxidation of metals on QD surfaces for improved photostability regardless of the reactivity or the $\Delta G_{\mathrm{f}}$ of the individual metal, even including some inactive or inert metals which are difficult to be oxidized spontaneously in an ambient atmosphere. In addition, our method can also be employed in the CIS/ZnS:Al QD system for significantly improved photostability (Fig. S17†). Therefore, these results demonstrated that our SOSMS strategy should be a versatile method to grow metal oxide layers on QD surfaces and improve their stability.

\section{Conclusions}

In summary, we have developed an effective approach to form a metal oxide passivation layer on individual QD by sacrificial oxidation of a self-metal source. By employing CdSe/CdS and CdSe/CdS:Al QDs, an appropriate peroxide (BPO) treatment can easily and rapidly convert the inherent metal (Cd and $\mathrm{Al})$ on the surface of QDs to metal oxides ( $\mathrm{CdO}$ and $\mathrm{Al}_{2} \mathrm{O}_{3}$ ). With this treatment, the photostability of QDs was greatly improved without damaging the optical properties. In addition, this method can be extended to other doped QD systems, such as CdSe/CdS:Cr, CdSe/CdS:Zr and CIS/ZnS:Al QDs. Principally, we believe that our approach is limited to neither QDs nor doping with $\mathrm{Al}$, which makes it a versatile strategy for other nanomaterials, such as metal nanoclusters $\left(\mathrm{Au}^{4}{ }^{44} \mathrm{Ag},{ }^{45}\right.$ or $\left.\mathrm{Cu}^{46}\right)$ and metal oxide nanoparticles $\left(\mathrm{Fe}_{3} \mathrm{O}_{4}{ }^{47}\right)$ for improved photostability and opens up new avenues in various practical applications, including in vivo imaging, lighting and displays, environmental monitoring, and chemical analysis.

\section{Conflicts of interest}

There are no conflicts of interest to declare. 


\section{Acknowledgements}

This work was financially supported by the National Key Research and Development Program of China (2016YFD0800207) and the National Natural Science Foundation of China (NSFC 21773155). We acknowledge Professor Zhengtao Deng from Nanjing University for performing the absolute PLQY measurement. We thank Professor Xiaogang Peng from Zhejiang University and Professor Rui Si from the Shanghai Institute of Applied Physics, CAS, for valuable discussions.

\section{References}

1 P. Michler, A. Kiraz, C. Becher, W. V. Schoenfeld, P. M. Petroff, L. Zhang, E. Hu and A. Imamoglu, Science, 2000, 290, 2282-2285.

2 P. Senellart, G. Solomon and A. White, Nat. Nanotechnol., 2017, 12, 1026-1039.

3 F. Hu, H. Zhang, C. Sun, C. Yin, B. Lv, C. Zhang, W. W. Yu, X. Wang, Y. Zhang and M. Xiao, ACS Nano, 2015, 9, 1241012416.

4 X. Dai, Z. Zhang, Y. Jin, Y. Niu, H. Cao, X. Liang, L. Chen, J. Wang and X. Peng, Nature, 2014, 515, 96-99.

5 V. L. Colvin, M. C. Schlamp and A. P. Alivisatos, Nature, 1994, 370, 354-357.

6 W. Cao, C. Xiang, Y. Yang, Q. Chen, L. Chen, X. Yan and L. Qian, Nat. Commun., 2018, 9, 2608.

7 C. M. Chuang, P. R. Brown, V. Bulović and M. G. Bawendi, Nat. Mater., 2014, 13, 796-801.

8 O. E. Semonin, J. M. Luther, S. Choi, H. Y. Chen, J. Gao, A. J. Nozik and M. C. Beard, Science, 2011, 334, 1530-1533.

9 X.-Y. Yu, B.-X. Lei, D.-B. Kuang and C.-Y. Su, Chem. Sci., 2011, 2, 1396-1400.

10 L. F. Shi, V. D. Paoli, N. Rosenzweig and Z. Rosenzweig, J. Am. Chem. Soc., 2006, 128, 10378-10379.

11 B. S. Shah, P. A. Clark, E. K. Moioli, M. A. Stroscio and J. J. Mao, Nano Lett., 2007, 7, 3071-3079.

12 M. A. Hines and P. G. Sionnest, J. Phys. Chem., 1996, 100, 468-471.

13 O. Chen, J. Zhao, V. P. Chauhan, J. Cui, C. Wong, D. K. Harris, H. Wei, H.-S. Han, D. Fukumura, R. K. Jain and M. G. Bawendi, Nat. Mater., 2013, 12, 445-451.

14 S. Jun and E. Jang, Angew. Chem., Int. Ed., 2013, 52, 679-682. 15 S. Huang, Z. Li, L. Kong, N. Zhu, A. Shan and L. Li, J. Am. Chem. Soc., 2016, 138, 5749-5752.

16 S. Cho, J. Kwag, S. Jeong, Y. Baek and S. Kim, Chem. Mater., 2013, 25, 1071-1077.

17 M. Adam, Z. Wang, A. Dubavik, G. M. Stachowski, C. Meerbach, Z. Soran-Erdem, C. Rengers, H. V. Demir, N. Gaponik and A. Eych müller, Adv. Funct. Mater., 2015, 25, 2638-2645.

18 H. Y. Kim, D.-E. Yoon, J. Jang, D. Lee, G.-M. Choi, J. H. Chang, J. Y. Lee, D. C. Lee and B.-S. Bae, J. Am. Chem. Soc., 2016, 138, 16478-16485.

19 Y. R. Do, D.-H. Park and Y.-S. Kim, J. Electrochem. Soc., 2004, 151, H210-H212.
20 S.-W. Seo, E. Jung, C. Lim, H. Chae and S. M. Cho, Appl. Phys. Express, 2012, 5, 035701.

21 R. R. Pareja, R. L. Ibáñez, F. Martín, J. R. Ramos-Barrado and D. Leinen, Surf. Coat. Technol., 2006, 200, 6606-6610.

22 K. B. Subila, G. K. Kumar, S. M. Shivaprasad and K. G. Thomas, J. Phys. Chem. Lett., 2013, 4, 2774-2779.

23 H. H. Uhlig and T. L. O'Connor, J. Electrochem. Soc., 1955, 102, 562-572.

24 V. M. Jovanovic and N. Hackerman, J. Phys. Chem. B, 1998, 102, 9855-9860.

25 R. J. Wilbraham, C. Boxall, D. T. Goddard, R. J. Taylor and S. E. Woodbury, J. Nucl. Mater., 2015, 464, 86-96.

26 W. E. Cass, J. Am. Chem. Soc., 1946, 68, 1976-1982.

27 C. Walling and J. Pellon, J. Am. Chem. Soc., 1957, 79, 47864788.

28 R. Tucker and S. Walton, Pharm. J., 2007, 279, 48-53.

29 R. Saravanan, M. M. Khan, V. K. Gupta, E. Mosquera, F. Gracia, V. Narayanan and A. Stephen, J. Colloid Interface Sci., 2015, 452, 126-133.

30 S. Anandan, N. Ohashi and M. Miyauchi, Appl. Catal., B, 2010, 100, 502-509.

31 I. Budai and G. Kaptay, Intermetallics, 2011, 19, 423-425.

32 Z. Li, W. Yao, L. Kong, Y. Zhao and L. Li, J. Am. Chem. Soc., 2015, 137, 12430-12433.

33 A. Fernandez-Perez, C. Navarrete, P. Valenzuela, W. Gacitua, E. Mosquera and H. Fernandez, Thin Solid Films, 2017, 623, 127-134.

34 J. K. Cooper, S. Gul, S. A. Lindley, J. Yano and J. Z. Zhang, ACS Appl. Mater. Interfaces, 2015, 7, 10055-10066.

35 G. E. McGuire, G. K. Schweitzer and T. A. Carlson, Inorg. Chem., 1973, 12, 2450-2453.

36 A. Shavorskiy, K. Müller, J. T. Newberg, D. E. Starr and H. Bluhm, J. Phys. Chem. C, 2014, 118, 29340-29349.

37 Y. F. Gao, M. Nagai, Y. Masuda, F. Sato, W. S. Seo and K. Koumoto, Langmuir, 2006, 22, 3521-3527.

38 N. Cai and G. Zhou, J. Phys. Chem. C, 2013, 117, 172-178.

39 S. C. Erwin, L. Zu, M. I. Haftel, A. L. Efros, T. A. Kennedy and D. J. Norris, Nature, 2005, 436, 91-94.

40 P. K. Jain, B. J. Beberwyck, L.-K. Fong, M. J. Polking and A. P. Alivisatos, Angew. Chem., Int. Ed., 2012, 51, 2387-2390.

41 J. Zhang, Q. Di, J. Liu, B. Bai, J. Liu, M. Xu and J. Liu, J. Phys. Chem. Lett., 2017, 8, 4943-4953.

42 A. Zavabeti, J. Z. Qu, B. J. Carey, N. Syed, R. Orrell-Trigg, E. L. H. Mayes, C. Xu, O. Kavehei, A. P. O'Mullane, R. B. Kaner, K. Kalantar-zadeha and T. Daeneke, Science, 2017, 358, 332-335.

43 Y.-Y. Chang, C.-P. Chang, D.-Y. Wang, S.-M. Yang and W. Wu, J. Alloys Compd., 2008, 461, 336-341.

44 Y. Shichibu, Y. Negishi, H. Tsunoyama, M. Kanehara, T. Teranishi and T. Tsukuda, Small, 2007, 3, 835-839.

45 Z. Shen, H. Duan and H. Frey, Adv. Mater., 2007, 19, 349-352. 46 K. P. Rice, E. J. Walker Jr, M. P. Stoykovich and A. E. Saunders, J. Phys. Chem. C, 2011, 115, 1793-1799.

47 M. Kim, Y. Chen, Y. Liu and X. Peng, Adv. Mater., 2005, 17, 1429-1432. 